\title{
Induction of the Phase II Detoxification Pathway Suppresses Neuron Loss in Drosophila Models of Parkinson's Disease
}

\author{
Kien Trinh, ${ }^{1}$ Katherine Moore, ${ }^{1}$ Paul D. Wes, ${ }^{4}$ Paul J. Muchowski, ${ }^{3}$ Joyoti Dey, ${ }^{2}$ Laurie Andrews, ${ }^{1}$ and Leo J. Pallanck ${ }^{1}$ \\ ${ }^{1}$ Department of Genome Sciences and ${ }^{2}$ Molecular and Cellular Biology Program, University of Washington, Seattle, Washington 98195 , ${ }^{3} \mathrm{Gladstone}$ Institute \\ of Neurological Disease and Departments of Biochemistry and Biophysics, and Neurology, University of California, San Francisco, San Francisco, California \\ 94158, ${ }^{4}$ Elan Pharmaceuticals, South San Francisco, California 94080
}

$\alpha$-Synuclein aggregates are a common feature of sporadic Parkinson's disease (PD), and mutations that increase $\alpha$-synuclein abundance confer rare heritable forms of PD. Although these findings suggest that $\alpha$-synuclein plays a central role in the pathogenesis of this disorder, little is known of the mechanism by which $\alpha$-synuclein promotes neuron loss or the factors that regulate $\alpha$-synuclein toxicity. To address these matters, we tested candidate modifiers of $\alpha$-synuclein toxicity using a Drosophila model of PD. In the current work, we focused on phase II detoxification enzymes involved in glutathione metabolism. We find that the neuronal death accompanying $\alpha$-synuclein expression in Drosophila is enhanced by loss-of-function mutations in genes that promote glutathione synthesis and glutathione conjugation. This neuronal loss can be overcome by genetic or pharmacological interventions that increase glutathione synthesis or glutathione conjugation activity. Moreover, these same pharmacological agents suppress neuron loss in Drosophila parkin mutants, a loss-of-function model of PD. Our results suggest that oxidative stress is a feature of $\alpha$-synuclein toxicity and that induction of the phase II detoxification pathway represents a potential preventative therapy for PD.

Key words: $\alpha$-synuclein; dopamine; Drosophila; synucleinopathy; glutathione; glutathione S-transferase

\section{Introduction}

Parkinson's disease (PD) is a common neurodegenerative disorder characterized by the loss of dopamine (DA) neurons in the midbrain and the accumulation of neuronal inclusions known as Lewy bodies (Dawson and Dawson, 2003). Although the involvement of Lewy bodies in the pathogenesis of PD is currently unclear, genetic studies of rare familial forms of PD have demonstrated that $\alpha$-synuclein protein, a principle component of Lewy body inclusions (Spillantini et al., 1997), is an active participant in the pathogenesis of this disorder (Polymeropoulos et al., 1997; Kruger et al., 1998; Zarranz et al., 2004). The precise biological function of $\alpha$-synuclein and the mechanism by which mutations in this gene confer neuron loss are currently unknown. However, the finding that $\alpha$-synuclein toxicity directly correlates with its gene dosage in several familial forms of this disease (Singleton et al., 2003; Chartier-Harlin et al., 2004) indicates that excess $\alpha$-synuclein protein can cause DA neuron loss (Cuervo et al.,

Received Jan. 22, 2007; accepted Nov. 19, 2007.

K.T. was supported in part by a Genetic Approaches to Aging Training Grant (5T32AG00057-28a), and P.J.M. was supported by the National Institute of Neurological Disease and Stroke (1R01NS047237). Additional support for this study was provided by grants to L.J.P. from the Michael J. Fox Foundation and the National Institutes of Health (1R01NS41780-01). We thank Dr. Helen Benes for providing us with a GstS1 null mutant, Dr. S. Birman for the TH-GAL4 transgenic line, and Dr. W. C. Orr for the UAS-GCIm transgenic line. We would also like to thank Dr. Thomas Montine, Aimee Schantz, and the University of Washington Alzheimer's Disease Research Center (Grant P50 AG05136) for providing human tissues, and Dr. Thomas Bird, Dr. James B. Leverenz, and Dr. Alex Whitworth for advice. Finally, we thank Amber Fechko and Adeena Khan for technical assistance.

Correspondence should be addressed to Dr. Leo J. Pallanck, University of Washington, 1705 NE Pacific Street, Foege S-443E, Box 355065, Seattle, WA 98195-5065. E-mail: pallanck@u.washington.edu.

DOI:10.1523/JNEUROSCI.4778-07.2008

Copyright $\odot 2008$ Society for Neuroscience $\quad 0270-6474 / 08 / 280465-08 \$ 15.00 / 0$
2004). Although mutations of the $\alpha$-synuclein gene are a rare cause of PD, the presence of $\alpha$-synuclein in the Lewy body inclusions that define both genetic and idiopathic forms of this disorder strongly suggests that this protein is a participant in most forms of PD.

In an effort to define the mechanism of $\alpha$-synuclein toxicity, a number of animal models of $\alpha$-synucleinopathy have been created through the expression of human $\alpha$-synuclein protein (Feany and Bender, 2000; Masliah et al., 2000; van der Putten et al., 2000; Auluck et al., 2002; Giasson et al., 2002; Lee et al., 2002; Lakso et al., 2003; Outeiro and Lindquist, 2003). Although these models have been useful in studies of candidate pathways of $\alpha$-synuclein toxicity, a yeast model of $\alpha$-synucleinopathy has also proven useful in the identification of novel genes and pathways that influence $\alpha$-synuclein toxicity. In particular, two recent genetic screens in yeast have led to the identification of 34 extragenic suppressors (Cooper et al., 2006) and $>100$ extragenic enhancers of $\alpha$-synuclein toxicity in this organism (Willingham et al., 2003; Cooper et al., 2006). However, only a small number of these factors have thus far been examined in a neuronal context.

In the current study, we have used a fly model of $\alpha$-synucleinopathy to examine a subset of the yeast $\alpha$-synuclein enhancers that appear to be involved in the phase II detoxification pathway, specifically, glutathione metabolism. Our studies demonstrate that loss-of-function mutations affecting glutathione synthesis or glutathione conjugation pathways enhance DA neuron loss in flies overexpressing human $\alpha$-synuclein. Moreover, overexpression of factors involved in glutathione biosynthesis and glutathione conjugation suppress $\alpha$-synuclein toxicity. Finally, we find that feeding $\alpha$-synuclein-expressing flies or Dro- 
sophila parkin mutants pharmacological inducers of the phase II detoxification pathway suppresses the neuronal loss of both of these models of PD. Our findings provide the foundation for a possible therapeutic strategy for PD.

\section{Materials and Methods}

Construction of the UAS-synuclein transgene. The $\alpha$-synuclein coding sequence was amplified from a wild-type (WT) human $\alpha$-synuclein cDNA using the following primer set: $5^{\prime}$ primer, CAACTCGAGACAAAATGGATGTATTCATGAAAGGAC; 3' primer, CAATCTAGATTAGGCTTCAGGTTCGTAGTCTTG. These primers include nucleotide alterations preceding the normal start codon in the $\alpha$-synuclein cDNA that are designed to improve translational efficiency in Drosophila. These $5^{\prime}$ and $3^{\prime}$ primer sets also include $X h o I$ and $X b a I$ restriction sites, respectively, to allow cloning of the cDNA into the Drosophila transformation vector pUAST (Brand and Perrimon, 1993). The final transformation construct was sequenced to confirm the absence of PCR-introduced errors and used to generate germline transformant flies using standard procedures.

Generation of Drosophila strains. The TH-GAL4, UAS-Gclm, and $U A S-G s t S 1$ transgenic lines and the GstS1 ${ }^{M 26}$ null allele have been previously described (Friggi-Grelin et al., 2003; Orr et al., 2005; Whitworth et al., 2005) All P-element lines used in this work, as well as the $\mathrm{Gclm}^{L 0580}$ mutant, were obtained from the Bloomington Drosophila stock center. Previous work has demonstrated that the recessive lethal phenotype associated with the chromosome bearing the $\mathrm{Gclm}^{L 0580}$ P-element insertion is conferred by an unrelated mutation residing on this chromosome. This recessive lethal mutation was removed by meiotic recombination to generate a homozygous viable $\mathrm{Gclm}^{L 0580}$ strain for this study. The CG4365 ${ }^{\Delta}$ allele was generated using a standard P-element imprecise excision protocol to delete $\sim 1 \mathrm{~kb}$ of the $5^{\prime}$ coding sequence of the CG4365 gene. The UAS-Syn ${ }^{L P 3}$ insertion situated on chromosome 3 was recombined with a TH-GAL4 insertion also situated on chromosome 3 , to generate a recombinant chromosome 3 bearing both of these insertions. The TH-GAL4 insertion situated on chromosome 3 was mobilized to chromosome 2 using a standard P-element transposition procedure, and this insertion was recombined with the UAS-Syn ${ }^{L P 2}$ insertion situated on chromosome 2 to generate a recombinant chromosome 2 bearing both of these insertions. All fly crosses were performed at $25^{\circ} \mathrm{C}$ on standard cornmeal/molasses food.

Western blot analysis. Protein extracts for Western blot analysis were prepared by homogenizing fly heads in lysis buffer (50 mM Tris, pH 7.4, $150 \mathrm{~mm} \mathrm{NaCl}, 0.1 \% \mathrm{NP}-40$ ) plus protease inhibitors. Lysates were then subjected to centrifugation for $10 \mathrm{~min}$ at $12,000 \times \mathrm{g}$ to remove cellular debris, and the protein concentration of the lysates were determined using a commercial Bradford assay (Bio-Rad, Hercules, CA). Ten micrograms of total protein from each sample were subjected to SDS-PAGE electrophoresis. After electrophoresis, proteins were transferred to nitrocellulose membrane and were probed with antisera to human $\alpha$-synuclein (LB509; 1:500 dilution, Millipore, Billerica, MA) and $\alpha$-tubulin (1:1000, Sigma-Aldrich, St. Louis, MO).

Analysis of DA neurons and glutathione abundance. Analysis of DA neuron integrity was performed as previously described by Whitworth et al. (2005). Briefly, aged flies were anesthetized and dissected to remove the entire brain. Brains were then fixed in $4 \%$ paraformaldehyde in $1 \times$ phosphosaline buffer $\left(10 \mathrm{~mm} \mathrm{Na}_{2} \mathrm{HPO}_{4}, 2 \mathrm{mM} \mathrm{KH}_{2} \mathrm{PO}_{4}, 0.14 \mathrm{M} \mathrm{NaCl}, 2.7\right.$ $\mathrm{mm} \mathrm{KCl}, \mathrm{pH}$ 7.4). After fixation, brain tissues were washed three times for 20 min each with wash buffer (PBS $+0.1 \%$ Triton X-100), then incubated in blocking buffer $(0.1$ Tris- $\mathrm{HCl}, \mathrm{pH} 7.5,0.15 \mathrm{NaCl}, 0.1 \%$ Triton $\mathrm{X}-100$, and $10 \% \mathrm{FBS}$ ) for $1 \mathrm{~h}$ at room temperature. Tissues were then incubated with anti-tyrosine hydroxylase antibody (1:100, Millipore) overnight at $4{ }^{\circ} \mathrm{C}$. This was followed by incubation with Alexa anti-rabbit secondary antibody (1:1000, Invitrogen, Carlsbad, CA) for $2 \mathrm{~h}$ at room temperature. Brains were washed three times for $20 \mathrm{~min}$ each in wash buffer and mounted on coverslips with Prolong Antifade media (Invitrogen). DA neurons were visualized by confocal microscopy using a BioRad MRC600 or an Olympus (Tokyo, Japan) FV-1000 confocal microscope. The number of DA neurons in each cluster was determined by visual inspection of the individual confocal $Z$-series. All analyses were performed with the experimenter blinded to genotype. For each experiment, the number of brains analyzed was $\geq 16$. Statistical significance was determined using ANOVA analysis.

Glutathione abundance in larval and cellular lysates was determined using a commercial glutathione detection kit (Millipore) according to the manufacturer's directions. All glutathione abundance measurements were performed at least three times. Two third instar larvae or $3 \times 10^{6}$ cells were used for each glutathione abundance measurement, and statistical significance was determined using the Student's $t$ test.

Drug feeding assay. Drug feeding experiments were performed by diluting a $37.5 \mathrm{mg} / \mathrm{ml}$ stock solution of DL-sulforaphane (Sigma-Aldrich) in DMSO and an $8 \mathrm{mg} / \mathrm{ml}$ stock solution of allyl disulfide (Sigma-Aldrich) in water directly into melted fly food. The final concentrations of sulforaphane and allyl disulfide in fly food were 25 and $30 \mu \mathrm{g} / \mathrm{ml}$, respectively. $\alpha$-Synuclein-expressing flies were collected after eclosion and placed on drug-treated food for $20 \mathrm{~d}$. Second instar parkin mutant larvae were raised on drug-treated food until eclosion, and the adult flies were transferred to fresh drug-treated food for $20 \mathrm{~d}$. To control for the possible influence of DMSO on the neuronal loss phenotype, control flies were placed on food containing an equivalent concentration of DMSO for the same period of time.

\section{Results}

Previous work has shown that expression of $\alpha$-synuclein in the Drosophila brain induces the loss of tyrosine hydroxylase (TH)positive neurons in the protocerebral posterior medial 1 (PPM1) and PPM2 clusters (Fig. $1 B$ ) in an age-dependent manner (Feany and Bender, 2000). These studies involved the use of TH immunohistochemical staining of paraffin-imbedded thin sections to document the loss of DA neurons. However, more recent studies of these same transgenic lines using confocal microscopy failed to detect loss of TH-positive neurons in any of the major DA neuron clusters in Drosophila (Auluck et al., 2005; Pesah et al., 2005). These conflicts appear to be explained by a reduction in $\mathrm{TH}$ expression in the PPM1 and PPM2 clusters of DA neurons in $\alpha$-synuclein transgenic flies and improved sensitivity of the confocal methodology in detecting $\mathrm{TH}$-positive neurons relative to light microscopy (Auluck et al., 2005).

Because several familial mutations that cause heritable forms of parkinsonism result from increased gene dosage of $\alpha$-synuclein (Singleton et al., 2003; Chartier-Harlin et al., 2004), we reasoned that increased expression of $\alpha$-synuclein in Drosophila might result in loss of TH-positive neurons that is detectable by confocal microscopy. To test this hypothesis, we generated an $\alpha$-synuclein expression construct bearing sequence alterations designed to improve the translational efficiency of this cDNA in Drosophila (see Materials and Methods for details). This modified $\alpha$-synuclein cDNA was placed downstream of a GAL4-responsive upstream activating sequence (UAS) element and introduced into the Drosophila germline. Several independent transgenic $U A S$-synuclein lines were obtained, and these lines were crossed to flies bearing the pan-neuronal elav-GAL4 driver to induce expression of $\alpha$-synuclein protein in the brain. Analysis of the expression levels of our newly generated transgenic lines indicates that they direct the production of twofold to fivefold higher levels of $\alpha$-synuclein protein than previously reported UAS-synuclein transgenic lines (Fig. $1 A$ ).

To maximize $\alpha$-synuclein protein expression in the fly brain, all of our experiments made use of flies bearing two copies of the $U A S$-synuclein transgenes and two copies of the GAL4 drivers, which approximately doubles the abundance of $\alpha$-synuclein protein relative to flies bearing a single copy of each of these transgenes (data not shown). Although these flies express more $\alpha$-synuclein protein than previously described fly models of $\alpha$-synucleinopathy, the abundance of $\alpha$-synuclein in the brains of 
A

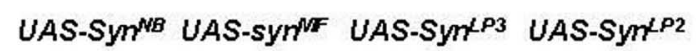
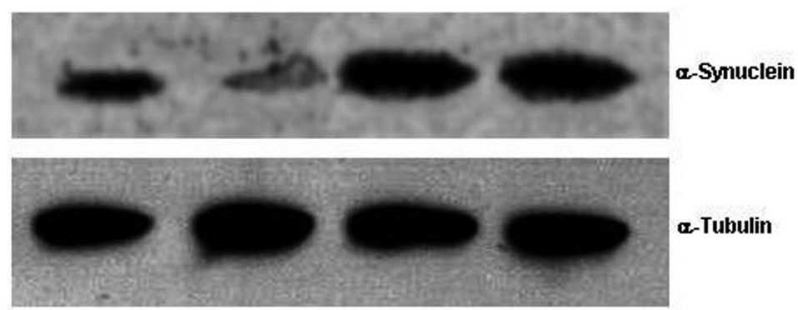

$\mathbf{F}$

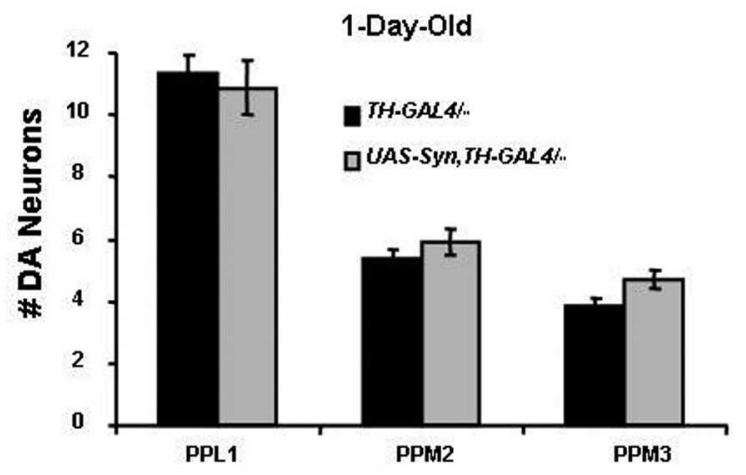

G

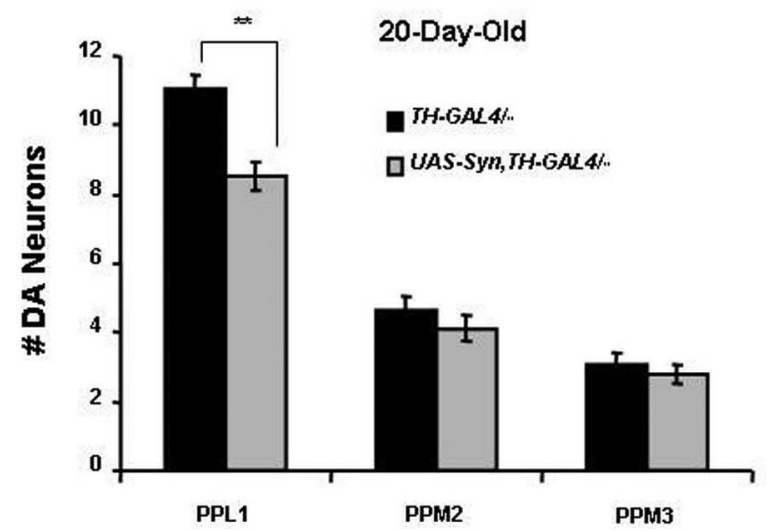

B
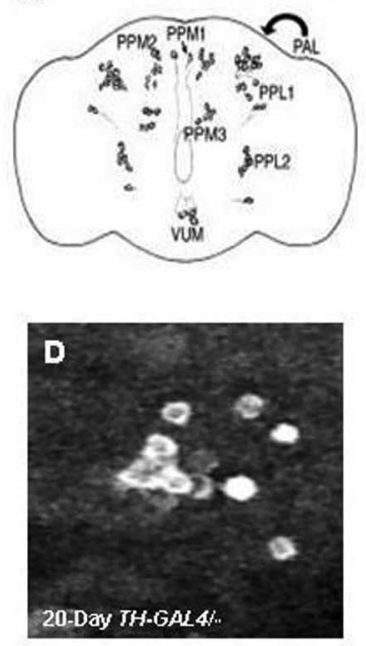
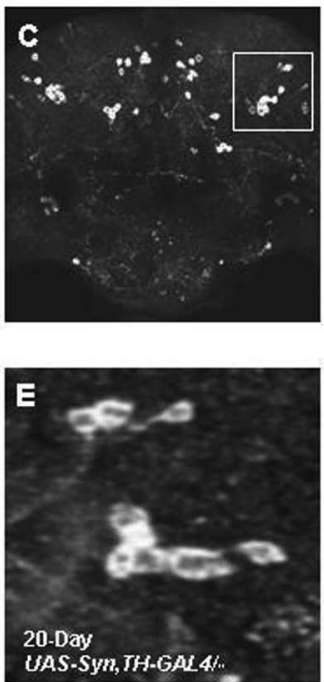

H

20-Day-Old

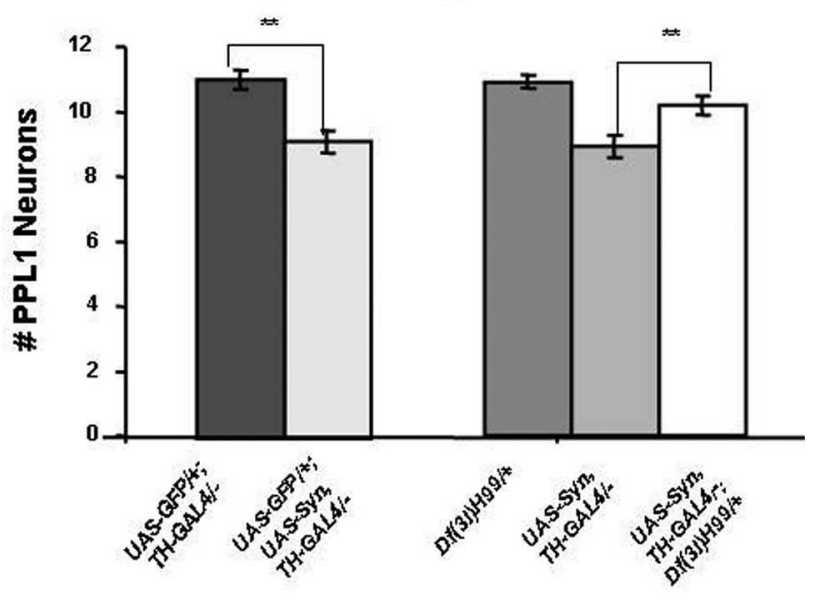

Figure 1. Abundant $\alpha$-synuclein expression causes progressive DA neuron loss. A, A Western blot comparing $\alpha$-synuclein protein abundance in the heads of flies bearing the pan-neuronal elav-GAL4 driver and one of four different UAS-synuclein transgenic lines. UAS-Syn ${ }^{N B}$ and UAS-Syn ${ }^{M F}$ are previously reported transgenic lines (Auluck et al., 2002) (Feany and Bender, 2000). $U A S-S y n^{L P 3}$ and UAS-Syn ${ }^{L P 2}$ are transgenic lines generated in the current study. Tubulin serves as a protein loading control. $B$, Schematic diagram showing some of the major DA neuron clusters in an adult fly brain. PAL, Protocerebral anterior lateral (anterior to PPL1 as indicated by arrow); VUM, ventral unpaired medial. C, Confocal image from a wild-type brain immunostained with anti-TH to mark DA neurons. The boxed area indicates the PPL1 cluster of DA neurons shown in higher magnification in $\boldsymbol{D}$ and $\boldsymbol{E}$. D, E, A representative PPL1 neuron cluster from a 20-d-old control fly bearing two copies of the TH-GAL4 driver (D) and a representative PPL1 neuron cluster in a 20-d-old fly bearing two copies of the TH-GAL4 driver and two copies of the UAS-Syn transgene (E). $\boldsymbol{F}, \boldsymbol{G}$, The number of DA neurons in three major DA neuron clusters of adult control flies homozygous for the TH-GAL4 transgene and of adult flies homozygous for two copies of the TH-GAL4 and UAS-Syn transgenes at $1 \mathrm{~d}$ of age $(\boldsymbol{F})$ and at $20 \mathrm{~d}$ of age $(\boldsymbol{G})$. $\alpha$-Synuclein-expressing flies have significant DA neuron loss restricted to the PPL1 cluster at $20 \mathrm{~d}$ of age $\left.{ }^{* *} p<0.005\right)$. $\boldsymbol{H}$, The neuron loss resulting from $\alpha$-synuclein expression is also detectable by comparing GFP fluorescence in flies bearing a UAS-GFP reporter in addition to two copies of the TH-GAL4 driver and in flies bearing a UAS-GFP transgene in addition to two copies of the UAS-Syn transgene and two copies of the TH-GAL4 driver. Introducing a copy of the Df(3L)H99 deletion, which removes several proapoptotic genes, suppresses neuron loss in flies bearing two copies of UAS-Syn and two copies of $T H$-GAL4 relative to flies lacking the Df(3L)H99 deletion. The Df(3L)H99 deficiency did not detectably influence neuronal integrity in a WT background.

flies bearing two copies of the elav-GAL4 driver and two copies of our UAS-synuclein transgenes is similar to that of the normal human neostriatum (supplemental Fig. S1, available at www. jneurosci.org as supplemental material). We detected no loss of TH-positive neurons in 1-d-old flies bearing multiple copies of the $\alpha$-synuclein transgenes and the GAL4 drivers relative to con- trols (Fig. $1 F$ and data not shown). In contrast, 20-d-old flies bearing two copies of the UAS-synuclein transgene and two copies of the TH-GAL4 driver had a statistically significant reduction in the number of TH-positive neurons in the protocerebral posterior lateral 1 (PPL1) cluster relative to WT controls (Fig. $1 E, G$ ). A similar pattern of neuronal loss was obtained using two copies 
of an independent UAS-synuclein transgene and two copies of an independent TH-GAL4 insertion (supplemental Fig. S2, available at www.jneurosci.org as supplemental material), indicating that the observed results are the direct result of $\alpha$-synuclein expression, and are unrelated to the insertion loci associated with the transgenes used in these experiments. However, no neuronal loss was detected in flies bearing one or two copies of the $\alpha$-synuclein and elav-GAL4 transgenes at 20 or more days of age, likely reflecting decreased efficiency of the elav-GAL4 driver relative to TH-GAL4 in TH-positive neurons (supplemental Figs. S1, S3, available at www.jneurosci.org as supplemental material).

The neuronal loss resulting from $\alpha$-synuclein expression by the TH-GAL4 driver can also be detected with an independent monitoring system involving GFP expression using a UAS-GFP transgene (Fig. $1 \mathrm{H}$ ), indicating that the observed loss of TH-positive neurons is not simply an artifact of poor antibody penetration or decreased TH abundance. Moreover, the $\alpha$-synuclein induced loss of TH-positive neurons in the PPL1 cluster was suppressed by introducing a chromosome bearing a deletion of the proapoptotic factors Reaper, Grim, and Hid (Fig. $1 \mathrm{H}$ ). Together, our findings indicate that abundant $\alpha$-synuclein expression in Drosophila results in progressive DA neuron cell death.

To explore the mechanism of $\alpha$-synuclein toxicity, we used our transgenic model of $\alpha$-synucleinopathy to test candidate enhancers of $\alpha$-synuclein toxicity that were identified in a yeast model of PD. Among the $86 \alpha$-synuclein enhancers identified in a previous study (Willingham et al., 2003), we selected 10 Drosophila genes for analysis (supplemental Table S1, available at www. jneurosci.org as supplemental material) using the following criteria: first, only genes with a single Drosophila ortholog and at least one human homolog were considered; second, only Drosophila genes with available corresponding mutations were considered for our initial analysis. Among these genes, we noted that three, GstS1, Eip55E, and CG4365, encode proteins that potentially influence glutathione metabolism. The GstS1 gene encodes a glutathione S-transferase, which catalyzes the ligation of reduced glutathione to a variety of substrates, including the toxic products of reactive oxygen species (Singh et al., 2001). The Eip55E gene encodes a putative cystathionine $\gamma$-lyase involved in the biosynthesis of cysteine, which is rate limiting for the production of glutathione (Lu, 1998). The uncharacterized gene, CG4365, encodes a putative hydroxyacylglutathione hydrolase activity that is involved in a glutathione salvage pathway. Given that the GstS1, Eip55E, and CG4365 genes may influence a single pathway, and that previous work implicates alterations in glutathione metabolism in the etiology of PD (Spina and Cohen, 1989; Sian et al., 1994; Bharath et al., 2002; Bharath and Andersen, $20 \mathrm{~d}$.
20-Day-Old

B

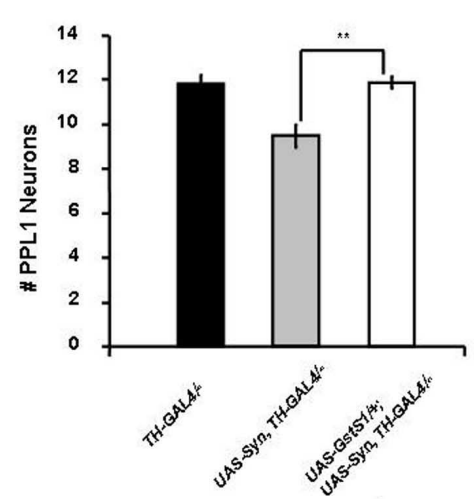

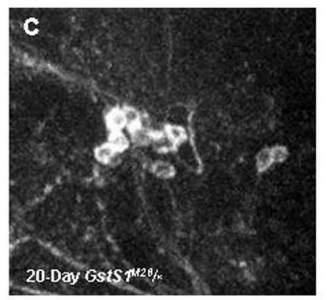
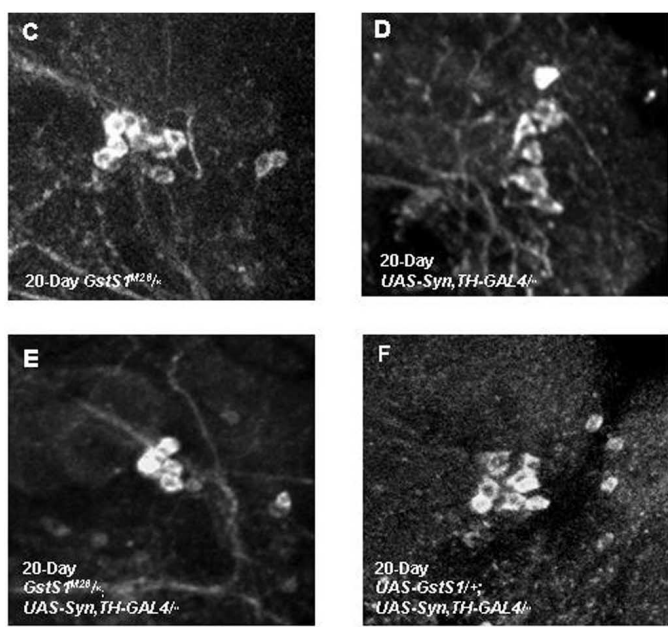

Figure 2. GstS1 activity modifies $\alpha$-synuclein-induced DA neuron loss. A, Twenty-day-old GstS $1^{M 26}$ homozygotes display no significant DA neuron loss relative to control flies. However, the DA neuron loss in the PPL1 cluster that is detected in 20-d-old flies bearing two copies of the UAS-Syn and TH-GAL4 transgenes is enhanced in 20-d-old flies that are also homozygous for the oss observed in 20-d-old flies bearing two copies of the UAS-Syn and TH-GAL4 transgenes (**p $<0.005)$. C-F Representative images showing the PPL1 DA neurons in GstS $7^{\text {M26 }}$ homozygotes $(\boldsymbol{C})$, flies bearing two copies of the UAS-Syn and TH-GAL4 transgenes (D), GstST ${ }^{M 26}$ homozygotes bearing two copies of the UAS-Syn and TH-GAL4 transgenes $(\boldsymbol{E})$, and flies bearing two copies of the UAS-Syn and TH-GAL4 transgenes and one copy of the UAS-GstS7 transgene $(\boldsymbol{F}) . \boldsymbol{C}-\boldsymbol{F}$ are from the brains of flies aged

2005), we chose to focus on these three genes in our current study.

Our previous work indicates that a null allele of the Drosophila GstS1 gene, GstS1 $1^{M 26}$, enhances the DA neuron loss and muscle degeneration phenotypes of Drosophila parkin mutants. Moreover, transgenic overexpression of GstS1 in Drosophila parkin mutants partially suppresses these phenotypes (Whitworth et al., 2005). Thus, we tested whether similar alterations in GstS1 gene dosage would influence the DA neuron loss phenotype of our Drosophila model of $\alpha$-synucleinopathy. To explore the effects of decreased GstS1 activity on $\alpha$-synuclein toxicity, we expressed $\alpha$-synuclein in a GstS1 null background using the GstS1 ${ }^{M 26}$ null allele. The GstS1 ${ }^{M 26}$ mutation was found to enhance the DA neuron loss phenotype of $\alpha$-synuclein-expressing flies relative to flies expressing $\alpha$-synuclein alone (Fig. $2 A, E$ ). This increased neuronal loss appears to be a synergistic effect of GstS1 mutations on $\alpha$-synuclein toxicity, because our previous work indicates that GstS1 ${ }^{M 26}$ mutants do not exhibit detectable neuronal loss (Whitworth et al., 2005), and these findings were independently confirmed in the present study (Fig. 2 A). To test whether overexpres- 

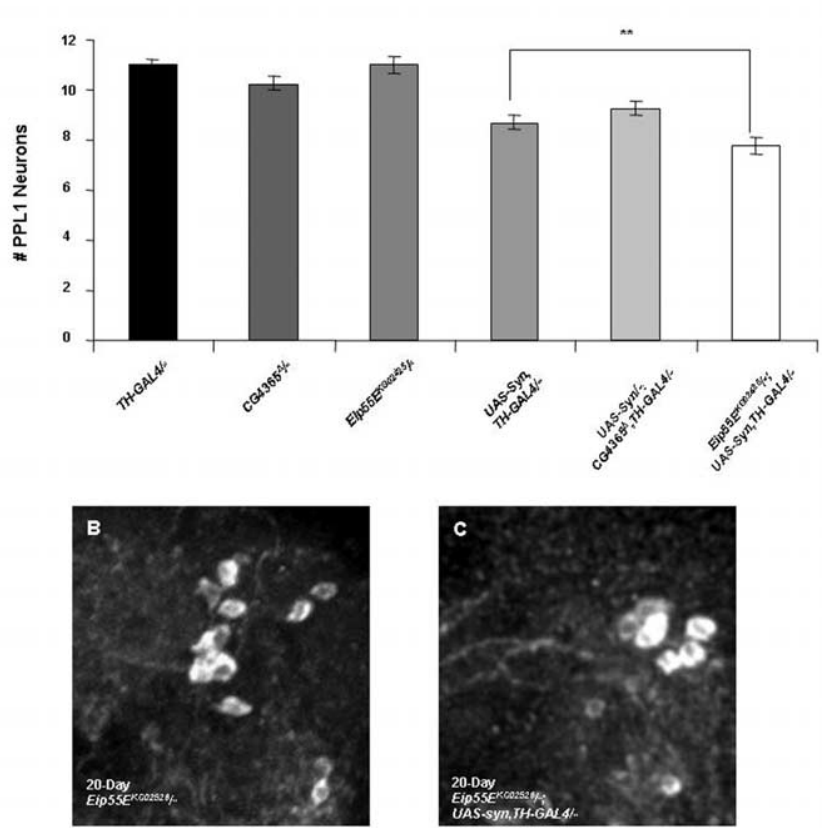

Figure 3. A hypomorphic mutation of the Eip55E gene enhances $\alpha$-synuclein-induced DA neuron loss. A, Eip55E ${ }^{K G 02526}$ homozygotes do not display detectable loss of DA neurons in the PPL1 cluster relative to flies bearing two copies of the TH-GAL4 driver. However, the DA neuron loss in the PPL1 cluster that is observed in 20-d-old flies bearing two copies of the UAS-Syn and TH-GAL4 transgenes is further reduced in flies homozygous for the Eip55E ${ }^{K G 02526}$ mutation $\left({ }^{* *} p<0.005\right)$. However, the neuronal loss seen in $\left(G 4365^{\Delta}\right.$ homozygotes bearing two copies of the UAS-Syn and TH-GAL4 transgenes is indistinguishable from that of WT flies bearing two copies of the UAS-Syn and TH-GAL4 transgenes. $B, C$, Representative images of PPL1 neurons from 20-d-old Eip55E ${ }^{K G 02526}$ homozygotes $(\boldsymbol{B})$ and 20-d-old Eip55E ${ }^{K G 02526}$ homozygotes bearing two copies of the UAS-Syn and TH-GAL4 transgenes (C).

sion of GstS1 is able to suppress the DA neuron loss phenotype of $\alpha$-synuclein-expressing flies, we generated adult flies bearing a $U A S-G s t S 1$ transgene, as well as two copies of the UAS-synuclein transgene and two copies of the TH-GAL4 driver. Analyses of these flies indicated that elevated GstS1 expression fully suppressed the neuronal loss observed in 20-d-old $\alpha$-synucleinexpressing flies (Fig. 2B,F). Together, these findings demonstrate that the abundance of GstS1 directly influences the toxicity of $\alpha$-synuclein protein in DA neurons.

Although our findings indicate that GstS1 activity is rate limiting in determining $\alpha$-synuclein toxicity, it is unclear from these findings whether alterations in glutathione abundance would also influence the toxicity of $\alpha$-synuclein. To test this possibility, we explored the influence of mutations in the Eip55E and CG4365 genes, which encode enzymes that potentially influence glutathione abundance. A homozygous viable P-element allele of the Eip55E gene that reduces Eip55E transcript abundance by $\sim 40 \%$ (data not shown) and an imprecise excision allele of CG4365 (see Materials and Methods), designated Eip55E ${ }^{K G 02526}$ and $C G 4365^{\Delta}$, respectively, were used in all subsequent analyses.

Analysis of DA neuron integrity in Eip55E $E^{K G 02526}$ homozygotes failed to reveal evidence of neuronal loss in 20-d-old flies, indicating that this mutation has no effect on DA neuron integrity in the absence of $\alpha$-synuclein expression (Fig. 3A). However, the loss of DA neurons caused by $\alpha$-synuclein expression in 20$\mathrm{d}$-old adults is enhanced by the Eip $55 E^{K G 02526}$ mutation (Fig. $3 A$ ). To test whether the effect of Eip55E $E^{K G 02526}$ on the toxicity of $\alpha$-synuclein is explained by the influence of this mutation on glutathione abundance, we assayed glutathione abundance in Eip55E $E^{K G 02526}$ homozygotes. This analysis revealed that glutathione abundance was decreased by approximately one-third in Eip55E $E^{K G 02526}$ homozygotes relative to a WT control (supplemental Fig. S4, available at www.jneurosci.org as supplemental material). In contrast, the $C G 4365^{\Delta}$ allele was found to have no effect on $\alpha$-synuclein toxicity or glutathione abundance (Fig. $3 \mathrm{~A}$ and data not shown).

Our studies of the Eip55E gene suggest that decreased glutathione abundance enhances the toxicity of $\alpha$-synuclein. However, because Eip55E presumably promotes the biosynthesis of cysteine, we cannot exclude the possibility that the enhanced toxicity of $\alpha$-synuclein in Eip55E mutants results instead from decreased cysteine abundance. To provide a more rigorous test of the hypothesis that alterations in glutathione abundance influence the toxicity of $\alpha$-synuclein, we sought a Drosophila mutant that would specifically affect glutathione biosynthesis. The ratelimiting enzyme in glutathione biosynthesis is glutamatecysteine lyase (Gcl) (Meister and Anderson, 1983). Although there are no mutations in the Drosophila gene that encodes this enzyme, previous work has led to the identification of mutations in the Drosophila Gclm gene, which encodes a Gcl-modifying subunit $(\mathrm{Gclm})$. In the current study, we used a homozygous viable, hypomorphic allele of $\mathrm{Gclm}$, designated $\mathrm{Gclm}^{\mathrm{LO580}}$, which confers a $45 \%$ reduction in glutathione abundance (Fraser et al., 2003). To assess the affects of altered glutathione abundance on $\alpha$-synuclein toxicity, we crossed the $G \mathrm{clm}^{L 0580}$ mutation, as well as available transgenic lines that overexpress the Gclm protein to $\alpha$-synuclein-expressing flies. As in the other experiments described in this study, these analyses were performed in flies that bear two copies of the UAS-synuclein and TH-GAL4 transgenes.

The decreased Gclm activity in $\mathrm{Gclm}^{\text {L0580 }}$ homozygotes was found to significantly enhance the DA neuron loss induced by $\alpha$-synuclein expression (Fig. 4A). In contrast, age-matched $G c l m^{L 0580}$ homozygotes in the absence of $\alpha$-synuclein expression exhibit no loss in DA neuron integrity, indicating that the neuronal effects of decreased glutathione levels are only manifest in flies overexpressing $\alpha$-synuclein. We also explored the effects of increased Gcl activity on $\alpha$-synuclein toxicity by using a $U A S-G c l m$ transgenic line previously reported to increase Gcl activity (Orr et al., 2005). Results of these analyses indicate that Gclm overexpression in DA neurons completely rescued the neuronal loss induced by $\alpha$-synuclein expression in 20- and 30-d-old flies (Fig. $4 B$ and data not shown). Additionally, overexpression of the UAS-Gclm transgene using the elav-GAL4 driver elevated glutathione abundance approximately twofold relative to controls (supplemental Fig. S4, available at www.jneurosci.org as supplemental material). Together, these findings indicate that the toxicity of $\alpha$-synuclein inversely correlates with the abundance of glutathione.

Both the Gclm and GstS1 genes are members of the phase II detoxification pathway, which promotes the conjugation of glutathione to a variety of electrophilic substrates. Previous work indicates that several naturally occurring chemical agents, including sulforaphane and allyl disulfide, are capable of inducing the phase II detoxification pathway by activating Nrf2, a key transcription factor that globally regulates this pathway. Drosophila also encodes an Nrf2 homolog, although the effects of sulforaphane and allyl disulfide on the induction of the phase II detoxification pathway have not previously been examined in flies. To explore the neuroprotective potential of these pharmacological agents, we first tested whether sulforaphane or allyl disulfide are able to induce the phase II detoxification pathway in Drosophila. 
We found that both of these chemical agents induced a significant increase in glutathione abundance in the Drosophila S2 cell line and in live animals, suggesting that the mechanism of action of sulforaphane and allyl disulfide is conserved in Drosophila (Fig. 5A-D). We next tested whether feeding $\alpha$-synuclein-expressing flies sulforaphane and allyl disulfide was capable of suppressing DA neuron loss. Because our previous work with Drosophila parkin mutants indicates that this model shares several features with our $\alpha$-synuclein model, including a modifying influence of altered glutathione metabolism on the parkin phenotypes, we also tested whether these chemical agents were capable of suppressing the neuron loss of this model. Remarkably, sulforaphane and allyl disulfide robustly suppressed neuronal loss in both of our models of PD (Fig. $5 E, F)$. Our findings raise the possibility that these and perhaps other chemical inducers of the phase II detoxification pathway represent potential preventative agents for PD.

\section{Discussion}

In an effort to generate a more robust Drosophila model of $\alpha$-synucleinopathy, we created novel $\alpha$-synuclein transgenic lines designed to increase the expression of $\alpha$-synuclein protein. These studies were prompted, in part, by recent genetic studies in humans showing that the toxicity of $\alpha$-synuclein correlates directly with $\alpha$-synuclein protein abundance (Eriksen et al., 2005). Although we were unable to detect any DA neuron loss in flies bearing a single copy of our $\alpha$-synuclein transgenes with any of the GAL4 drivers tested, flies bearing two copies of our $\alpha$-synuclein transgenes and two copies of the TH-GAL4 driver exhibit a progressive reduction of TH-positive neurons. This neuron loss is readily detected using two different confo-

A
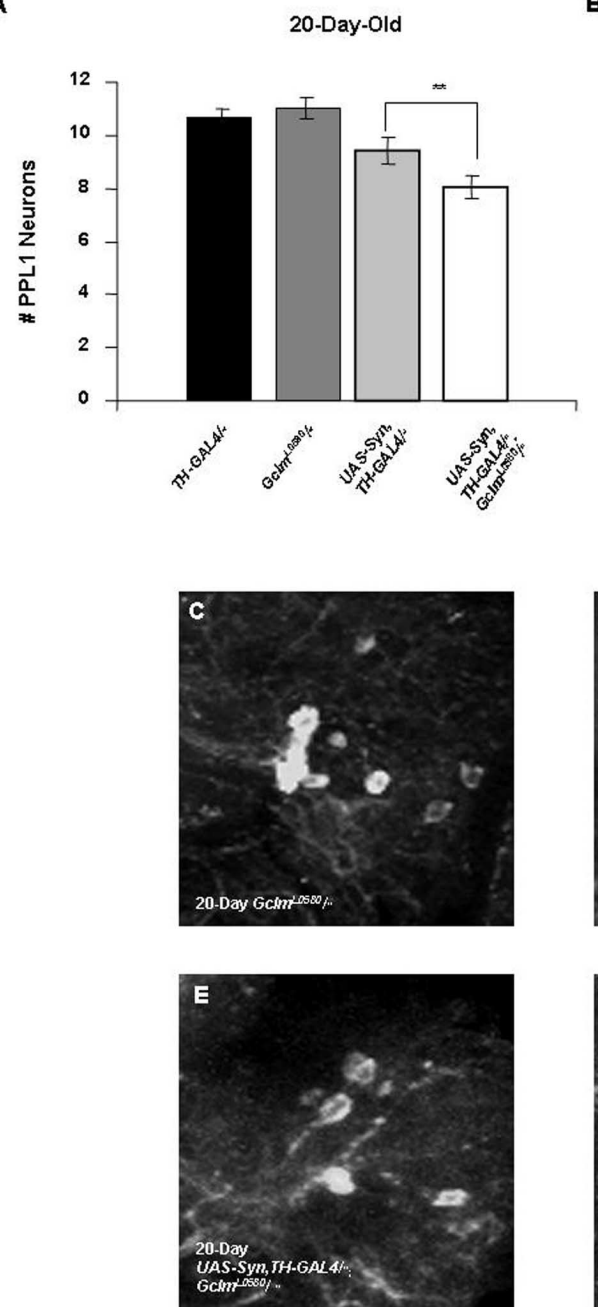
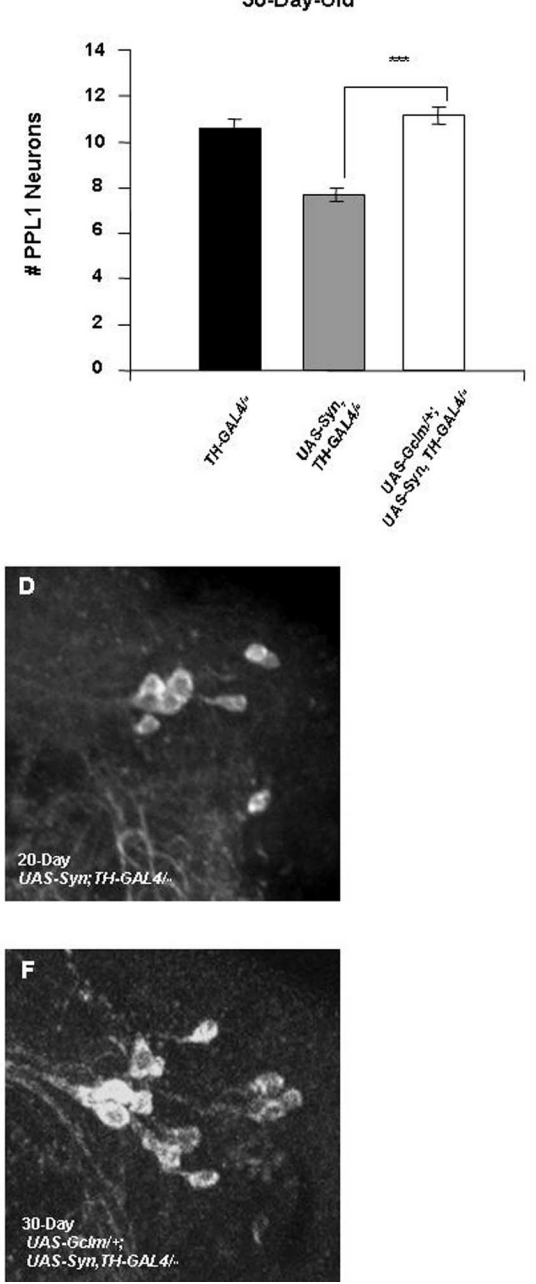

Figure 4. Geneticalterations directly affecting glutathione synthesis influence $\alpha$-synuclein toxicity. $A$, The number of neurons in the PPL1 cluster of 20-d-old GCIm ${ }^{20580}$ homozygotes is unaffected relative to control flies homozygous for the TH-GALL driver. However, 20-d-old Gclm ${ }^{L 0580}$ homozygotes bearing two copies of the UAS-Syn and TH-GAL4 transgenes have significantly fewer DA neurons in the PPL1 cluster relative to WT flies bearing two copies of the UAS-Syn and TH-GAL4 transgenes $\left({ }^{* *} p<0.005\right)$. $B$, Overexpression of GcIm using a UAS-GCIm transgene completely suppresses the neuron loss induced by two copies of the UAS-Syn and TH-GAL4 transgenes in 30-d-old adult brains ( $\left.{ }^{* * *} p<0.001\right) .(-F$, Representative confocal images showing PPL1 neurons in

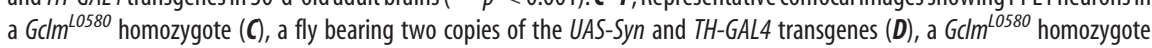
bearing two copies of the UAS-Syn and TH-GAL4 transgenes $(\boldsymbol{E})$, and a fly bearing two copies of the UAS-Syn and TH-GAL4 transgenes and a single copy of the UAS-GCIm transgene $(\boldsymbol{F}) . \boldsymbol{C}-\boldsymbol{E}$ are from the brains of flies aged $20 \mathrm{~d}$, and $\boldsymbol{F}$ is from the brain of a fly aged $30 \mathrm{~d}$.

cal microscopic reporter systems, can be suppressed by reduced gene dosage of proapoptotic factors, and is conferred by independent insertions of the TH-GAL4 driver and UAS-synuclein transgenes. Together these findings indicate that abundant expression of $\alpha$-synuclein in Drosophila results in the death of a subpopulation of DA neurons through a cell-autonomous mechanism.

Surprisingly, the neuron loss in our model of $\alpha$-synucleinopathy is restricted to the PPL1 cluster, in contrast to previous work, which has reported neuron loss, or reduced $\mathrm{TH}$ staining, primarily restricted to the PPM1 and PPM2 clusters (Whitworth et al., 2006). However, because our studies did not discriminate between neurons that are weakly or strongly $\mathrm{TH}$ positive, we cannot exclude an effect of our transgenes on $\mathrm{TH}$ abundance in these or other DA neuron clusters. The variance in our studies also leaves open the possibility of a small degree of neurodegeneration in these clusters that is undetectable by our methodology.
The abundant $\alpha$-synuclein expression required for detectable cell loss in our model raises the possibility that this cell loss is simply a nonspecific consequence of foreign protein overexpression. Although this possibility is a necessary caveat of at least most dominant gain-of-function models of disease, several arguments suggest that the neuron loss in our model results from a relatively specific toxic feature of $\alpha$-synuclein. First, we do not detect obvious toxicity from $\alpha$-synuclein expression in other parts of the fly brain (including most DA neuron clusters), or in nonneuronal tissues using a number of different GAL4 drivers (supplemental Figs. S1, S3 and data not shown). Second, flies coexpressing $\alpha$-synuclein and GFP did not exhibit a more severe phenotype than flies expressing $\alpha$-synuclein alone (Fig. 1), suggesting that toxicity is not simply related to the total burden of foreign protein expression. Third, the PPL1 cluster of DA neurons that are sensitive to $\alpha$-synuclein is the same neuronal cluster that is most sensitive to loss-of-function mutations in the Dro- 

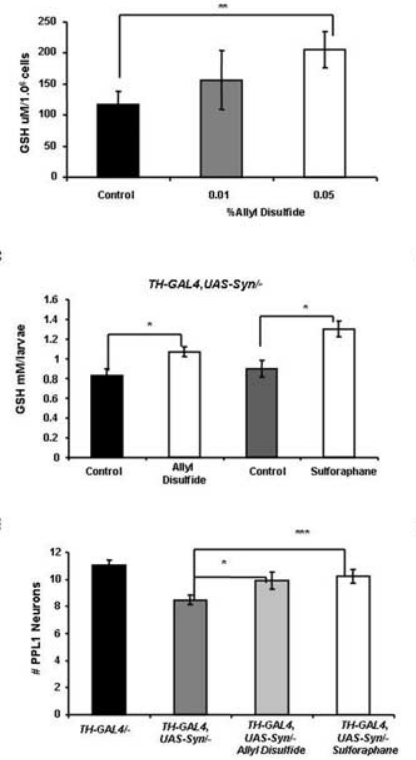
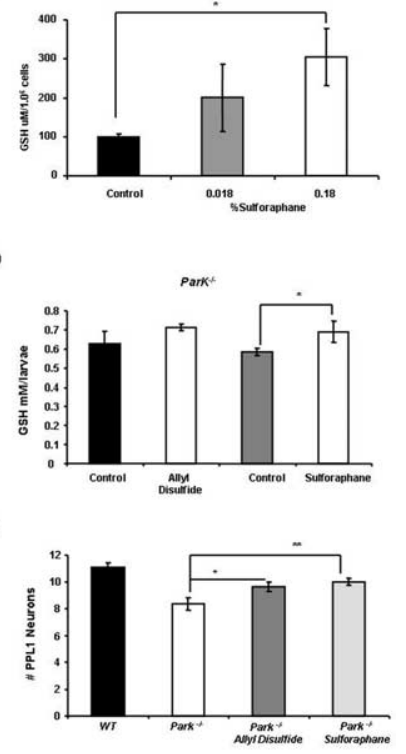

Figure 5. Pharmacological agents that induce the phase II detoxification pathway rescue neuronal loss in two different Drosophila models of Parkinson's disease. $A, B$, S2 cells treated for $24 \mathrm{~h}$ with allyl disulfide $(\boldsymbol{A})$ or DL-sulforaphane $(\boldsymbol{B})$ display a dose-dependent increase in glutathione abundance $\left({ }^{*} p<0.05 ;{ }^{* *} p<0.005\right)$. C, D, Larvae bearing two copies of the UAS-Syn and $T H$-GAL4 transgenes $(\boldsymbol{C})$ or that bear homozygous mutations of the park ${ }^{25}$ null allele $(\boldsymbol{D})$ also exhibit increased glutathione abundance when exposed to $30 \mu \mathrm{g} / \mathrm{ml}$ allyl disulfide or $25 \mu \mathrm{g} / \mathrm{ml}$ DL-sulforaphane for $4 \mathrm{~d}\left({ }^{*} p<0.05\right)$. To control for the possible influence of DMSO on glutathione abundance, control larvae were raised on food containing an equivalent concentration of DMSO for the same period of time. $\boldsymbol{E}$, Twenty-day-old flies bearing two copies of the UAS-Syn and $T H$-GAL4 transgenes that were raised on standard food supplemented with either 30 $\mu \mathrm{g} / \mathrm{ml}$ allyl disulfide $\left({ }^{*} p<0.05\right.$ ) or $25 \mu \mathrm{g} / \mathrm{ml}$ DL-sulforaphane $\left(^{* * *} p<0.01\right.$ ) exhibited substantially decreased neuronal loss relative to untreated flies. $\boldsymbol{F}$, Twenty-day-old Drosophila park $^{25}$ homozygotes raised on standard food supplemented with either $30 \mu \mathrm{g} / \mathrm{ml}$ allyl disulfide $\left({ }^{*} p<0.05\right)$ or $25 \mu \mathrm{g} / \mathrm{ml}$ DL-sulforaphane $\left({ }^{* * *} p<0.01\right)$ also exhibited decreased neuronal loss relative to untreated flies. Statistical analysis was performed using Student's $t$ test.

sophila parkin gene (Whitworth et al., 2005), suggesting that $\alpha$-synuclein expression confers a toxic feature that is common to other genetic perturbations responsible for PD in humans. Fourth, our finding that alterations in glutathione and glutathione-S transferase abundance influence $\alpha$-synuclein toxicity is consistent with previous literature implicating this pathway in the pathogenesis of PD (Spina and Cohen, 1989; Sian et al., 1994; Bharath and Andersen, 2005), and is less readily explained by a nonspecific toxic effect of foreign protein accumulation.

The inverse relationship between $\alpha$-synuclein toxicity and glutathione transferase activity/glutathione abundance suggests several potential models by which $\alpha$-synuclein may induce loss of DA neurons. One model that is consistent with the known role of glutathione in protection from oxidative stress is that overexpression of $\alpha$-synuclein induces oxidative stress. This model is supported by previous work on $\alpha$-synuclein showing that expression of either wild-type or mutant $\alpha$-synuclein protein increases intracellular reactive oxygen species levels (Junn and Mouradian, 2002). Another plausible model is that oxidative damage of $\alpha$-synuclein protein triggers its conversion to a toxic derivative. This potential model is supported by the finding that nitratively modified $\alpha$-synuclein is widespread in PD brains (Giasson et al., $2000)$ and that exposure of $\alpha$-synuclein-expressing cells to oxidative or nitrative agents induces the formation of $\alpha$-synuclein inclusions (Norris et al., 2003). Finally, it is also possible that $\alpha$-synuclein overexpression somehow triggers the depletion of

glutathione, or that alteration in glutathione metabolism influence the abundance of $\alpha$-synuclein protein or the general health of DA neurons. Although the reduced glutathione content of Drosophila parkin mutants (Fig. 5 and data not shown) raises the possibility that glutathione depletion is the ultimate cause of neuron loss in parkin mutants, we detected no obvious change in the glutathione content of flies overexpressing $\alpha$-synuclein relative to WT controls (data not shown), suggesting that this is an unlikely explanation for the toxicity of $\alpha$-synuclein overexpression. Moreover, we were unable to detect an effect of altered GstS1 or $\mathrm{Gcl}$ activity on $\alpha$-synuclein abundance (supplemental Fig. S5, available at www.jneurosci.org as supplemental material), and we detected no ill effects of reducing glutathione abundance or glutathione transferase activity in the absence of $\alpha$-synuclein. Experiments are currently underway to distinguish potential models by which glutathione metabolism influences $\alpha$-synuclein toxicity.

Our genetic findings demonstrating that induction of particular phase II detoxification pathway components are protective in fly models of PD were mimicked by chemical inducers of this pathway. Although further work will be required to define the specific phase II detoxification pathway component(s) most responsible for the neuroprotective effects of sulforaphane and allyl disulfide, our finding that these agents suppress neuron loss in two very different Drosophila models of PD, coupled with the fact that these phase II pathway inducers also work in a vertebrate context, suggests a promising treatment strategy for PD.

\section{References}

Auluck PK, Meulener MC, Bonini NM (2005) Mechanisms of suppression of $\alpha$-synuclein neurotoxicity by geldanamycin in Drosophila. J Biol Chem 280:2873-2878.

Auluck PK, Chan HY, Trojanowski JQ, Lee VM, Bonini NM (2002) Chaperone suppression of alpha-synuclein toxicity in a Drosophila model for Parkinson's disease. Science 295:865-868.

Bharath S, Andersen JK (2005) Glutathione depletion in a midbrainderived immortalized dopaminergic cell line results in limited tyrosine nitration of mitochondrial complex I subunits: implications for Parkinson's disease. Antioxid Redox Signal 7:900-910.

Bharath S, Hsu M, Kaur D, Rajagopalan S, Andersen JK (2002) Glutathione, iron and Parkinson's disease. Biochem Pharmacol 64:1037-1048.

Brand AH, Perrimon N (1993) Targeted gene expression as a means of altering cell fates and generating dominant phenotypes. Development 118:401-415.

Chartier-Harlin MC, Kachergus J, Roumier C, Mouroux V, Douay X, Lincoln S, Levecque C, Larvor L, Andrieux J, Hulihan M, Waucquier N, Defebvre L, Amouyel P, Farrer M, Destee A (2004) Alpha-synuclein locus duplication as a cause of familial Parkinson's disease. Lancet 364:1167-1169.

Cooper AA, Gitler AD, Cashikar A, Haynes CM, Hill KJ, Bhullar B, Liu K, Xu K, Strathearn KE, Liu F, Cao S, Caldwell KA, Caldwell GA, Marsischky G, Kolodner RD, Labaer J, Rochet JC, Bonini NM, Lindquist S (2006) Alpha-synuclein blocks ER-Golgi traffic and Rab1 rescues neuron loss in Parkinson's models. Science 313:324-328.

Cuervo AM, Stefanis L, Fredenburg R, Lansbury PT, Sulzer D (2004) Impaired degradation of mutant alpha-synuclein by chaperone-mediated autophagy. Science 305:1292-1295.

Dawson TM, Dawson VL (2003) Molecular pathways of neurodegeneration in Parkinson's disease. Science 302:819-822.

Eriksen JL, Przedborski S, Petrucelli L (2005) Gene dosage and pathogenesis of Parkinson's disease. Trends Mol Med 11:91-96.

Feany MB, Bender WW (2000) A Drosophila model of Parkinson's disease. Nature 404:394-398.

Fraser JA, Kansagra P, Kotecki C, Saunders RD, McLellan LI (2003) The modifier subunit of Drosophila glutamate-cysteine ligase regulates catalytic activity by covalent and noncovalent interactions and influences glutathione homeostasis in vivo. J Biol Chem 278:46369-46377.

Friggi-Grelin F, Coulom H, Meller M, Gomez D, Hirsh J, Birman S (2003) Targeted gene expression in Drosophila dopaminergic cells using regulatory sequences from tyrosine hydroxylase. J Neurobiol 54:618-627. 
Giasson BI, Duda JE, Murray IV, Chen Q, Souza JM, Hurtig HI, Ischiropoulos H, Trojanowski JQ, Lee VM (2000) Oxidative damage linked to neurodegeneration by selective alpha-synuclein nitration in synucleinopathy lesions. Science 290:985-989.

Giasson BI, Duda JE, Quinn SM, Zhang B, Trojanowski JQ, Lee VM (2002) Neuronal alpha-synucleinopathy with severe movement disorder in mice expressing A53T human alpha-synuclein. Neuron 34:521-533.

Junn E, Mouradian MM (2002) Human alpha-synuclein over-expression increases intracellular reactive oxygen species levels and susceptibility to dopamine. Neurosci Lett 320:146-150.

Kruger R, Kuhn W, Muller T, Woitalla D, Graeber M, Kosel S, Przuntek H, Epplen JT, Schols L, Riess O (1998) Ala30Pro mutation in the gene encoding alpha-synuclein in Parkinson's disease. Nat Genet 18:106-108.

Lakso M, Vartiainen S, Moilanen AM, Sirvio J, Thomas JH, Nass R, Blakely RD, Wong G (2003) Dopaminergic neuronal loss and motor deficits in Caenorhabditis elegans overexpressing human alpha-synuclein. J Neurochem 86:165-172.

Lee MK, Stirling W, Xu Y, Xu X, Qui D, Mandir AS, Dawson TM, Copeland NG, Jenkins NA, Price DL (2002) Human alpha-synuclein-harboring familial Parkinson's disease-linked Ala-53 $\rightarrow$ Thr mutation causes neurodegenerative disease with alpha-synuclein aggregation in transgenic mice. Proc Natl Acad Sci USA 99:8968-8973.

Lu SC (1998) Regulation of hepatic glutathione synthesis. Semin Liver Dis 18:331-343.

Masliah E, Rockenstein E, Veinbergs I, Mallory M, Hashimoto M, Takeda A, Sagara Y, Sisk A, Mucke L (2000) Dopaminergic loss and inclusion body formation in alpha-synuclein mice: implications for neurodegenerative disorders. Science 287:1265-1269.

Meister A, Anderson ME (1983) Glutathione. Annu Rev Biochem 52:711-760.

Norris EH, Giasson BI, Ischiropoulos H, Lee VM (2003) Effects of oxidative and nitrative challenges on alpha-synuclein fibrillogenesis involve distinct mechanisms of protein modifications. J Biol Chem 278:27230-27240.

Orr WC, Radyuk SN, Prabhudesai L, Toroser D, Benes JJ, Luchak JM, Mockett RJ, Rebrin I, Hubbard JG, Sohal RS (2005) Overexpression of glutamate-cysteine ligase extends life span in Drosophila melanogaster. J Biol Chem 280:37331-37338.

Outeiro TF, Lindquist S (2003) Yeast cells provide insight into alphasynuclein biology and pathobiology. Science 302:1772-1775.

Pesah Y, Burgess H, Middlebrooks B, Ronningen K, Prosser J, Tirunagaru V, Zysk J, Mardon G (2005) Whole-mount analysis reveals normal numbers of dopaminergic neurons following misexpression of alphaSynuclein in Drosophila. Genesis 41:154-159.
Polymeropoulos MH, Lavedan C, Leroy E, Ide SE, Dehejia A, Dutra A, Pike B, Root H, Rubenstein J, Boyer R, Stenroos ES, Chandrasekharappa S, Athanassiadou A, Papapetropoulos T, Johnson WG, Lazzarini AM, Duvoisin RC, Di Iorio G, Golbe LI, Nussbaum RL (1997) Mutation in the alphasynuclein gene identified in families with Parkinson's disease. Science 276:2045-2047.

Sian J, Dexter DT, Lees AJ, Daniel S, Agid Y, Javoy-Agid F, Jenner P, Marsden CD (1994) Alterations in glutathione levels in Parkinson's disease and other neurodegenerative disorders affecting basal ganglia. Ann Neurol 36:348-355.

Singh SP, Coronella JA, Benes H, Cochrane BJ, Zimniak P (2001) Catalytic function of Drosophila melanogaster glutathione S-transferase DmGSTS1-1 (GST-2) in conjugation of lipid peroxidation end products. Eur J Biochem 268:2912-2923.

Singleton AB, Farrer M, Johnson J, Singleton A, Hague S, Kachergus J, Hulihan M, Peuralinna T, Dutra A, Nussbaum R, Lincoln S, Crawley A, Hanson M, Maraganore D, Adler C, Cookson MR, Muenter M, Baptista M, Miller D, Blancato J, et al. (2003) alpha-Synuclein locus triplication causes Parkinson's disease. Science 302:841.

Spillantini MG, Schmidt ML, Lee VM, Trojanowski JQ, Jakes R, Goedert M (1997) Alpha-synuclein in Lewy bodies. Nature 388:839-840.

Spina MB, Cohen G (1989) Dopamine turnover and glutathione oxidation: implications for Parkinson disease. Proc Natl Acad Sci USA 86:1398-1400

van der Putten H, Wiederhold KH, Probst A, Barbieri S, Mistl C, Danner S, Kauffmann S, Hofele K, Spooren WP, Ruegg MA, Lin S, Caroni P, Sommer B, Tolnay M, Bilbe G (2000) Neuropathology in mice expressing human $\alpha$-synuclein. J Neurosci 20:6021-6029.

Whitworth AJ, Theodore DA, Greene JC, Benes H, Wes PD, Pallanck LJ (2005) Increased glutathione S-transferase activity rescues dopaminergic neuron loss in a Drosophila model of Parkinson's disease. Proc Natl Acad Sci USA 102:8024-8029.

Whitworth AJ, Wes PD, Pallanck LJ (2006) Drosophila models pioneer a new approach to drug discovery for Parkinson's disease. Drug Discov Today 11:119-126.

Willingham S, Outeiro TF, DeVit MJ, Lindquist SL, Muchowski PJ (2003) Yeast genes that enhance the toxicity of a mutant huntingtin fragment or alpha-synuclein. Science 302:1769-1772.

Zarranz JJ, Alegre J, Gomez-Esteban JC, Lezcano E, Ros R, Ampuero I, Vidal L, Hoenicka J, Rodriguez O, Atares B, Llorens V, Gomez Tortosa E, del Ser T, Munoz DG, de Yebenes JG (2004) The new mutation, E46K, of alphasynuclein causes Parkinson and Lewy body dementia. Ann Neurol 55: $164-173$. 